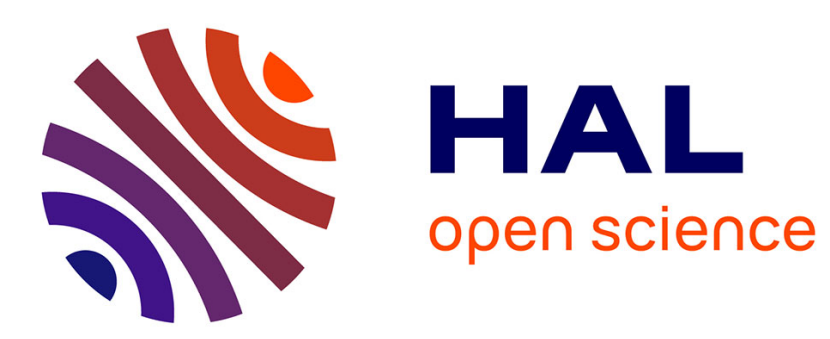

\title{
Solutions of slowly and periodically varying differential equations. Applications to some classical examples
} Nadir Sari

\section{To cite this version:}

Nadir Sari. Solutions of slowly and periodically varying differential equations. Applications to some classical examples. 2020. hal-02506680

\author{
HAL Id: hal-02506680 \\ https://hal.science/hal-02506680 \\ Preprint submitted on 12 Mar 2020
}

HAL is a multi-disciplinary open access archive for the deposit and dissemination of scientific research documents, whether they are published or not. The documents may come from teaching and research institutions in France or abroad, or from public or private research centers.
L'archive ouverte pluridisciplinaire HAL, est destinée au dépôt et à la diffusion de documents scientifiques de niveau recherche, publiés ou non, émanant des établissements d'enseignement et de recherche français ou étrangers, des laboratoires publics ou privés. 


\title{
SOLUTIONS OF SLOWLY AND PERIODICALLY VARYING DIFFERENTIAL EQUATIONS. APPLICATIONS TO SOME CLASSICAL EXAMPLES
}

\author{
NADIR SARI ${ }^{1}$
}

\begin{abstract}
We study a singularly perturbed second-order differential equation describing a slowly and periodically varying hamiltonian system. Typical dynamics governed by this type of system are, for example, equations of forced pendulum, of Duffing or of the "shallow water sloshing" problem. Using symmetries of this equation and singular perturbation tools, we describe dynamics, by splitting the phase space in regions where the motion is oscillatory and others where it is unbounded, and study dynamics in each kind of regions. Finally we establish the existence periodic solutions and give the structure of these solutions in term of response curves. In particular, our results extend and complete the ones stated in $[1,4,6]$ and answer to some open questions within. We also give new results about multiplicity of periodic solutions of forced pendulum equation. To illustrate our results, we conclude this work by a numerical study of these classical examples.
\end{abstract}

key words: singular perturbation, fast oscillations, periodic solutions, nonlinear resonance.

\section{InTRODUCTION}

We consider a slowly and periodically oscillating system described by the differential equation

$$
\frac{d^{2} x}{d \tau^{2}}=f(\varepsilon \tau, x)
$$

where $\varepsilon$ is a positive and small real parameter and $f$ is a $C^{1}$ function that is $2 \pi$-periodic and even function in its first variable.

\footnotetext{
${ }^{1}$ address: Laboratoire des Sciences de l'Ingénieur pour l'Environnement (LaSIE UMR CNRS 7356), Université de La Rochelle, 17042 La Rochelle, France E-mail: nsari@univ-lr.fr

2000 Mathematics Subject Classification. 34C25, 34B15, 34C23.
} 
After the change of time $t=\varepsilon \tau$, equation (1.1) becomes

$$
\varepsilon^{2} \frac{d^{2} x}{d t^{2}}=f(t, x)
$$

which is a singularly perturbed equation.

Many physical or mechanical phenomena are described by such equation. Classical examples of (1.1) are the equation of the forced pendulum

$$
\varepsilon^{2} \frac{d^{2} x}{d t^{2}}+\sin x=\beta \cos t
$$

Duffing equation

$$
\varepsilon^{2} \frac{d^{2} x}{d t^{2}}+a x+b x^{3}=\beta \cos t
$$

or equation of the "shallow water sloshing" problem

$$
\varepsilon^{2} \frac{d^{2} x}{d t^{2}}=x^{2}-(1+\lambda+\cos t)
$$

This last equation describing the motion of water in a rectangular tank forced to oscillate horizontally and periodically was first studied in [11] by numerical and asymptotical methods, were the authors gave various numerical results suggesting existence of many periodic solutions.

In fact, these equations often exhibit very complicated dynamics, like nonlinear resonance, subharmonics, period dubbing and chaos. For example in [11], Ockendon et al. have seen, as $\varepsilon$ decreases, more and more $2 \pi$-periodic solutions appear classified by the number $n$ of spikes near $t=0$ and zero or one spike near $t=\pi$. This phenomenon is also present in dynamics of pendulum and Duffing equations and many other examples [14]. In the case where equation (1.2) is even in time $t$, this kind of symmetry allows us to use a geometric method of shooting to find even $2 \pi$-periodic solutions. This method was introduced in [18], used in $[15,17]$ numerically and theoretically, and motivated by the fact that a great number of physical processes have internal symmetry properties and are modeled by differential equations, like (1.2), having this symmetry property: Since the second member of equation (1.2) is even in the time $t$, it is easy to see that every trajectory $\left(x(t), \frac{d x}{d t}(t)\right)$ starting at time $t=0$ from the point $\left(x_{0}, 0\right)$ is symmetric with respect to the $x$-axis (which we denote in the sequel by $x$-symmetric). Now, if there is $x_{0} \in \mathbb{R}$ such that $\frac{d x}{d t}(k \pi)=0$, then the corresponding trajectory is $2 k \pi$-periodic and the component $x(t)$ is an even $2 k \pi$-periodic solution of equation (1.2). Independently, a method of shooting was 
also used in [6] to study equation (1.5).

Figure 1 shows response curves of equation (1.3) with $\beta=0.3$, equation (1.4) with $a=1, b=-1 / 3, \beta=0.3$ and equation (1.5) with $\lambda=5$. These response curves are initial conditions with respect to $\omega=1 / \varepsilon$ of even $2 \pi$-periodic solutions. One can see different branches born near some particular values $\omega_{n}$. The corresponding values $\varepsilon_{n}$ are called nonlinear resonance values of the frequency $[10,19]$. Each branch of the response curves is a family $\left(\xi_{n, i}\right)$ of initial conditions of an even $2 k \pi$ periodic solution which has $n \in \mathbb{N}$ oscillations near $t=0$ and $i \in\{0,1\}$ spike near $t=\pi$. These branches are, for large $\omega$ asymptotic to values denoted $x_{s}$ and $x_{\sigma}$. One can see also that between to consecutive values of $\omega_{n}$ there is an isolated initial condition of periodic solution close to a value denoted $x_{c}$.

The goal of this paper is to give sufficient conditions of existence of periodic solutions for equation (1.2). This result allows us to explain the structure of the branches observed in figure 1 . In one hand, our results retrieve and complete the results stated in $[1,4,6]$, especially, by giving an answer to conjectures in $[1,6]$ concerning existence of fast oscillating periodic trajectories and existence of isolated trajectory near the center of oscillation. These kinds of solutions are no more attempted by the results in [4]. We discuss also the organization of these branches of solutions in term of bifurcation diagram when the parameter $\omega$ crosses non linear resonant values $\omega_{n}$. In the other hand, our results shed new light on existence and multiplicity of periodic solutions of pendulum equation (1.3) for small frequencies. This question of existence and multiplicity of periodic solutions especially for pendulum equation continue to mobilize a large number of researchers, the reader can find in $[8,9]$ a historical review of this problem.

The paper is organized as follows. We state in the next section, the main results of this work. we also study in this section the phase space of the equation (1.2); we split it in two regions, one region where the motion is oscillatory and another region where the motion is unbounded. In section 3, we describe the oscillatory motion and provide a condition of existence of oscillating trajectory defined for all $t$. We introduce the notion of number of rotations and study monotonicity of this number with respect to the initial condition $x_{0}$. Finally we study the motion near the center of oscillation and compute the nonlinear resonant values. In section 4 , we prove the main theorems of this paper. We conclude this work, with section 5, where we apply our results to equations (1.3), (1.4) and (1.5) and, to illustrate our results, provide 
(a)

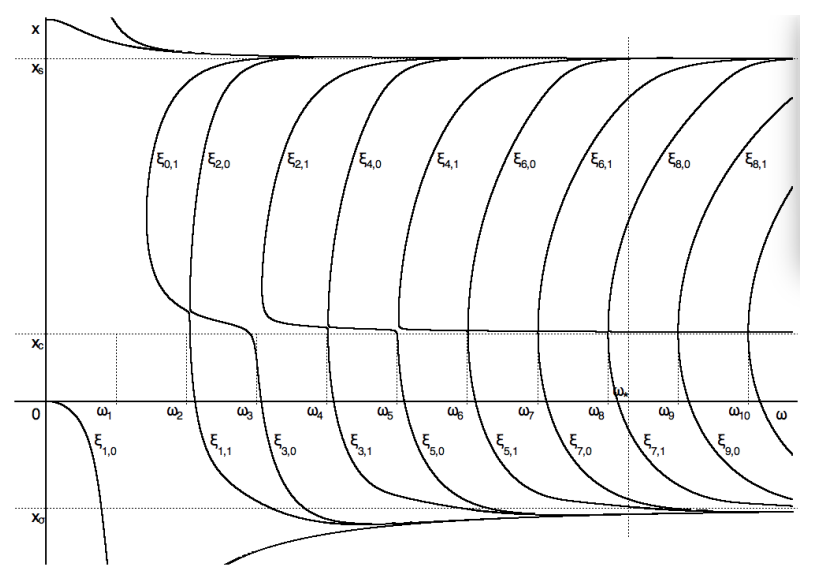

(b)

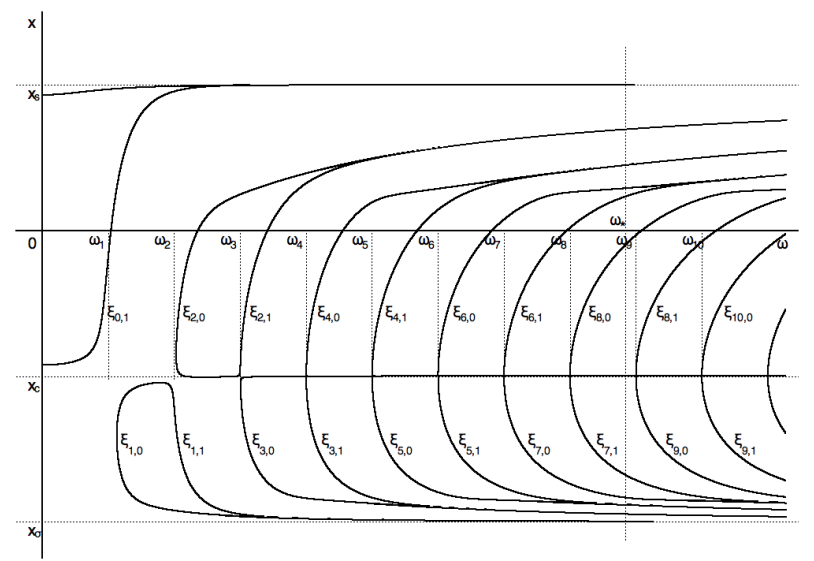

(c)

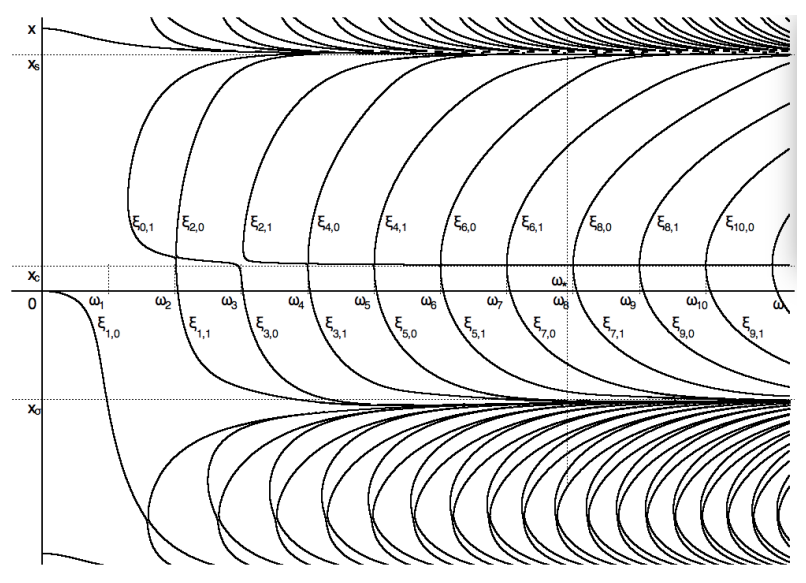

Figure 1. Response curves of (a) Duffing equation, (b) shallow water sloshing problem, (c) forced pendulum 
a numerical study of equation (1.5).

\section{Preliminaries and main Results}

Before stating the main results of this work, let us introduce some notations and definitions and also make some hypothesis. We consider the following dynamical system associated to equation (1.2)

$$
\left\{\begin{array}{l}
\varepsilon \frac{d x}{d t}=y \\
\varepsilon \frac{d y}{d t}=f(t, x)
\end{array}\right.
$$

Let $\bar{t} \in \mathbb{R}$, for small variation of $t$ around $\bar{t}$ of order $\varepsilon$, solutions of system (2.1) are approximated by solutions of the following autonomous dynamical system

$$
\left\{\begin{array}{l}
\frac{d x}{d T}=y \\
\frac{d y}{d T}=f(\bar{t}, x)
\end{array}\right.
$$

To see that it suffices to apply the change of time

$$
T=\frac{t-\bar{t}}{\varepsilon}
$$

System (2.1) becomes

$$
\left\{\begin{array}{l}
\frac{d x}{d T}=y \\
\frac{d y}{d T}=f(\bar{t}+\varepsilon T, x)
\end{array}\right.
$$

which is a regular perturbation of system (2.2) parametrized by $\bar{t}$. Now we introduce the following hypothesis

Hypothesis 2.1. For all $\bar{t} \in \mathbb{R}, f(\bar{t}, x)=0$ admits two isolated roots $x_{c}(\bar{t})$ and $x_{s}(\bar{t})$ such that $\frac{\partial f}{\partial x_{\bar{t}}}\left(\bar{t}, x_{c}(\bar{t})\right)<0$ and $\frac{\partial f}{\partial x}\left(\bar{t}, x_{s}(\bar{t})\right)>0$

This means that, for each $\bar{t} \in \mathbb{R}$, system (2.2) admits two stationary points:

$c(\bar{t})=\left(x_{c}(\bar{t}), 0\right)$ which is a center and $s(\bar{t})=\left(x_{s}(\bar{t}), 0\right)$ which is a saddle with a homoclinic separatrix enclosing $c(\bar{t})$.

Definition 2.1. The bounded region in $\mathbb{R}^{3}$ denoted by $O$ delimited by the family of homoclinic separatrices parametrized by $\bar{t}$ of the system (2.2) is called region of oscillatory motion.

$c(\bar{t})$ and $s(\bar{t})$, parametrized by $\bar{t}$, are called respectively center line and saddle line.

As long as $T$ is bounded (i.e. $t$ is close to $\bar{t}$ ) trajectories of system (2.3) are approximated by trajectories of system (2.2). Hence, at each 
time $\bar{t}$, one can describe, for small variation of $t$ around $\bar{t}$, the behavior of trajectories of system (2.1) by considering the phase portrait of system (2.2). System (2.2) admits the energy integral

$$
E(\bar{t}, x, y)=y^{2}+F(\bar{t}, x)
$$

where $F(\bar{t}, x)$ is the primitive

$$
F(\bar{t}, x)=-2 \int_{x_{c}(\bar{t})}^{x} f(\bar{t}, \xi) d \xi
$$

As long as $t=\bar{t}+\mathrm{O}(\varepsilon)$, the trajectory $\gamma(t)=(x(t), y(t))$ of system (2.1) starting at the time $\bar{t}$ from the point $(\bar{x}, \bar{y})$ is approximated by

$$
E(\bar{t}, x, y)=\bar{u}
$$

where $\bar{u}=E(\bar{t}, \bar{x}, \bar{y})$.

According to the value of the energy level $\bar{u}$, (i.e. the initial conditions $(\bar{t}, \bar{x}, \bar{y})$ ), an integral curve of system (2.2) defined by (2.6) may be a closed orbit, the homoclinic separatrix or an unbounded curve. Hence a trajectory of (2.1), as long as $t=\bar{t}+\mathrm{O}(\varepsilon)$, has three possible behaviors: It is approximated by a closed orbit, or by the homoclinic separatrix or by an unbounded trajectory of system (2.2).

When the variation of $t$ is no longer small, a trajectory of system (2.1) may have the three successive behaviors: an oscillatory motion, then approaches the homoclinic orbit of (2.2) and then follows an unbounded trajectory of (2.2).

The periodicity and symmetries of system (2.1) lead to periodicity and symmetries of its dynamics, it is easy to verify that $(0, x, y)$ and $(\pi, x, y)$ are symmetry planes. With these symmetries and periodicity, we can restrict the study of the phase space of $(2.1)$ to the interval of time $[0, \pi]$.

Definition 2.2. The unbounded region $\Omega$ of system (2.1) is the domain of $\mathbb{R}^{3}$ such that for all $(\bar{t}, \bar{x}, \bar{y}) \in \Omega$, the solution of system $(2.2)$ starting at $T=0$ from $(\bar{x}, \bar{y})$ is an unbounded trajectory.

Figure 2 illustrates the region of oscillatory motion $O$ and the region of unbounded motion $\Omega$. These two regions are separated by the surface denoted $S$ and constituted by the family of homoclinic separatrices parametrized by $\bar{t}$. We remark on this figure the important role played by equilibria of system (2.2) in the structure of dynamics of system (2.1). We denote by $\sigma(\bar{t})=\left(x_{\sigma}(\bar{t}), 0\right)$ the intersection the homoclinic separatrix at the time $\bar{t}$ with the plan $y=0$.

Definition 2.3. A trajectory $\gamma(t)=(x(t), y(t))$ of system (2.1) has a fast oscillating phase if there exists a nonempty interval $I$ such that for 


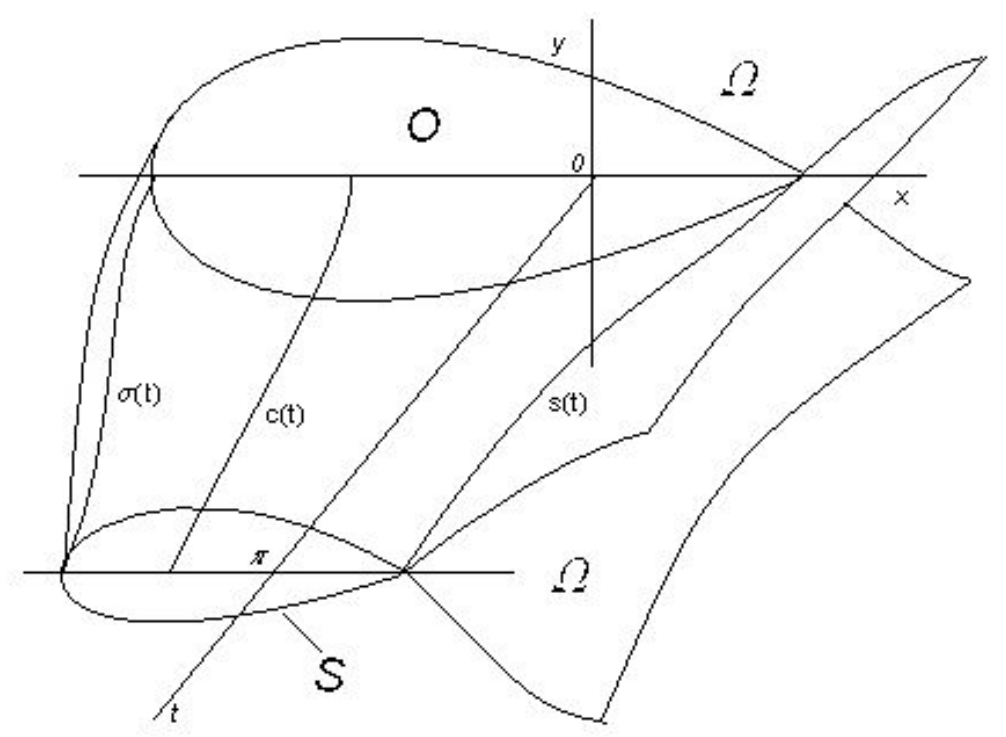

FiguRE 2. The region of oscillatory motion $O$ and the region of unbounded motion $\Omega$ separated by the surface $S$.

all $t$ in $I, \gamma(t)$ remains in the oscillatory region $O$. If $I=\mathbb{R}, \gamma(t)$ is called fast oscillating trajectory.

We introduce now the following notations:

Hypothesis 2.1 insures the existence of the region of oscillatory motion for all $t \in \mathbb{R}$. Indeed, for all $\bar{t} \in \mathbb{R}$, stationary points $c(\bar{t})$ and $s(\bar{t})$ are isolated and the center point is enclosed by the homoclinic separatrix of the saddle point. Let $\mathcal{A}(\bar{t})$ be the area of the surface delimited by this homoclinic separatrix. There exist $t_{*}$ and $t *$ in $[0, \pi]$ such that

$$
\forall \bar{t} \in[0, \pi] \mathcal{A}\left(t^{*}\right) \geq \mathcal{A}(\bar{t}) \geq \mathcal{A}\left(t_{*}\right)>0
$$

Let $u_{*}$ the real number such that the area of the surface delimited by the closed orbit of system (2.2) with $\bar{t}=0$ defined by equation $E(0, x, y)=u_{*}$ is equal to $\mathcal{A}\left(t_{*}\right)$.

Denote by $x_{i}\left(0, u_{*}\right), i=1,2$, the unique roots in the interval $\left(x_{\sigma}(0), x_{s}(0)\right)$ of equation $u_{*}-F(0, x)=0$.

We have, by definition of $u_{*}$ and $x_{i}\left(0, u_{*}\right)$,

$$
\mathcal{A}\left(t_{*}\right)=2 \int_{x_{1}\left(0, u_{*}\right)}^{x_{2}\left(0, u_{*}\right)} \sqrt{u_{*}-F(0, x)} d x
$$


Let constants $B_{*}$ and $C_{*}$ given by

$$
B_{*}=2 \int_{x_{1}\left(0, u_{*}\right)}^{x_{2}\left(0, u_{*}\right)} \frac{d x}{\sqrt{u_{*}-F(0, x)}}
$$

and

$$
C_{*}=\frac{1}{\pi} \int_{0}^{\pi} \sqrt{-\frac{\partial f}{\partial x} f\left(t, x_{c}(t)\right)} d t
$$

For all $\varepsilon>0, N_{\varepsilon}$ and $n_{\varepsilon}$ are respectively the integer part

$$
N_{\varepsilon}=\left[\frac{C_{*}}{\varepsilon}\right] \quad n_{\varepsilon}=\left[\frac{1}{B_{*} \varepsilon}\right]
$$

and $\left(\varepsilon_{n}\right)_{n>0}$ the sequence defined by $\varepsilon_{n}=\frac{C_{*}}{n}$.

Finally, we suppose this last hypothesis

Hypothesis 2.2. let $\Psi((t, x)$ the function defined by

$$
\Psi(t, x)=\frac{F(t, x)}{(f(t, x))^{2}}
$$

We suppose for each fixed $t \in \mathbb{R}$, the function $\Psi(t, x)$ is convex with respect to variable $x$.

We recall that a trajectory $\gamma(t)=(x(t), y(t))$ of system (2.1) starting from a point $\left(x_{0}, 0\right)$ at time $t=0$ is said $x$-symmetric if it is symmetric with respect to the $x$-axis that is if $\gamma(-t)=(x(t),-y(t))$. Hence $x(t)$ is an even solution of equation (1.2). Now we can state our main results.

Theorem 2.1. Let $a \in\left(0, C_{*} / 2\right)$ and define $\hat{\varepsilon}_{n}=\varepsilon_{n+1}\left(1+a \varepsilon_{n+1}\right)$ and $\bar{\varepsilon}_{n}=\varepsilon_{n}\left(1-a \varepsilon_{n}\right)$.

There exists $n_{0} \in \mathbb{N}$ such that for all integer $n>n_{0}$ and for all $\varepsilon \in\left(\hat{\varepsilon}_{n}, \bar{\varepsilon}_{n}\right)$, system (2.1) admits an unique $x$-symmetric $2 \pi$-periodic trajectory remaining in a tubular neighborhood of radius $\mathrm{O}(\varepsilon)$ of the center line $c(t)$.

Theorem 2.2. There exists $\delta>0$ such that for all $\varepsilon \in(0, \delta)$ and all integer $n$ such that $n_{\varepsilon} \leq n \leq N_{\varepsilon}$ system (2.1) possesses a $x$-symmetric $2 \pi$-periodic fast oscillating trajectory $\gamma_{n}(t)$ where $n$ is the number of oscillations by period $2 \pi$ around the center line $c(t)$.

Theorem 2.3. There exists $\delta>0$ such that for all $\varepsilon \in(0, \delta)$ system (2.1) possesses, for all $n \leq N_{\varepsilon}$ and $i \in\{0,1\}$, a x-symmetric $2 \pi$ periodic trajectory $\varphi_{n, i}(t)=\left(\xi_{n, i}(t), \zeta_{n, i}(t)\right)$ with a fast oscillating phase defined in a neighborhood of $t^{*}$, where $n$ is the number of oscillations performed during the fast oscillating phase and $i$ the number of spike at $t_{*}$, such that, $\xi_{n, i}\left(t_{*}\right)$ is close to $x_{s}\left(t_{*}\right)$ if $n$ is even and $\xi_{n, i}\left(t_{*}\right)$ is close to $x_{\sigma}\left(t_{*}\right)$ if $n$ is odd. 
Remark 2.1. Theorem 2.3 establishes the existence of trajectories (in our case periodic and symmetric) connecting hyperbolic invariant mani-folds for differential systems with slowly varying phase plane. Indeed, trajectories corresponding to small values of integer $p$ perform $p$ oscillations in an infinitesimal time of order $\mathrm{O}(\varepsilon)$ near time $t^{*}$, then spent the remaining time of the period in the vicinity of the saddle line $s(t)$ and may have 0 or 1 spike at time $t_{*}$.

This result can be connected to those obtained in [6] by shooting and in [4] by geometric singular perturbation (GSP) theory initiated by N. Fenichel in [3] and developed later by many authors in $[4,7,12,20]$ which contains many references. Particularly in [4] we can found a study of equation (1.5) by GSP methods.

Theorem 2.1, theorem 2.3 and theorem 2.2 retrieve and complete the results stated in $[4,6]$ by answering to some conjectures stated in $[1,6]$ about existence of rapidly oscillating periodic solution (theorem 2.2) and the existence of a solution near the center of oscillation $c(t)$ (Theorem 2.1). In [1], at page 391, the authors precise in the comments about equation (1.5) that they are not considering resonancephenomena. Theorem 4.9, at page 417, is analogue to my theorem 2.3 but in theorem 4.9 authors use also the symmetry of cosine at $t=\pi / 2$. In remark 4.11, at page 418, authors suppose the possibility to calculate an estimate of the number of periodic solutions in the oscillatory region. This estimate, constante $N_{\varepsilon}$, is established in theorem 2.3. Moreover, theorem 2.2 provides a lower bound, constante $n_{\varepsilon}$, of the number of $2 \pi$-periodic and fast oscillating solutions. This kind of solutions are not attempted by theorem 4.9 .

In there concluding remarks of [6], at page 245, authors write that they observe numerically existence of many solutions uniformly close to $y=-S(t)$, witch correspond in this work to center line $c(t)$, and are not covered by their results. In fact, in theorem 2.1, if $\varepsilon$ is far from the resonance values $\varepsilon_{n}$ there is a unique periodic trajectory in the vicinity of the center line.

The method of symmetries requires a good knowledge of the geometric behavior of trajectories. Since $\varepsilon$ is a small parameter, when $\tau$ has a bounded variation, $f(\varepsilon \tau, x)$ has a small variation then the differential system (2.3) has an "almost" first integral. Hence trajectories of this system follow the level curves of this "almost" first integral. When the level curves are closed, trajectories oscillate rapidly and may have a little drift along the nearby closed level curves. When the level curves are unbounded, trajectories escape, during a small time $t$ of order $O(\varepsilon)$, 
from the bounded phase space along these unbounded level curves. To make precise this heuristic description of this phenomenon for small parameter $\varepsilon$, we suppose that parameter $\varepsilon$ tends to 0 and use tools of singular perturbation theory. The main tool here is a method of averaging which describes the oscillatory dynamics by providing adiabatic invariants for oscillating trajectory and also allows to compute the number of rotations (oscillations) of this kind of trajectory around the center line $c(t)$.

\section{The Oscillatory MOTion}

Theorem of adiabatic invariance of the action of Hamiltonian system slowly varying in time applied to (2.1) has the following consequence

Proposition 3.1. ([16]) Let $\gamma(t)=(x(t), y(t))$ be the trajectory of system (2.1) starting from $\left(x_{0}, y_{0}\right)$ at $t_{0}$ such that the point $\left(t_{0}, x_{0}, y_{0}\right)$ is in the region of oscillatory motion $O$.

Let $\Gamma$ the surface given by

$$
E(t, x, y)=u(t)
$$

where $u(t)$ is the solution of the differential problem

$$
\left\{\begin{array}{l}
\frac{d u}{d t}=g(t, u) \\
u\left(t_{0}\right)=E\left(t_{0}, x_{0}, y_{0}\right)
\end{array}\right.
$$

with

$$
g(t, u)=\frac{\int_{x_{1}(t, u)}^{x_{2}(t, u)} \frac{\frac{\partial F}{\partial t}(t, x)}{\sqrt{u-F(t, x)}} d x}{\int_{x_{1}(t, u)}^{x_{2}(t, u)} \frac{d x}{\sqrt{u-F(t, x)}}}
$$

and $x_{i}(t, u), i=1,2$, are the roots of equation $u-F(t, x)=0$. Then, as long as $\gamma(t)$ belongs to $O$, it verifies

$$
E(t, x(t), y(t))=u(t)+O(\varepsilon)
$$

So one can see the cylindrical surface $\Gamma$ as an approximation of the graph of the trajectory $\gamma(t)$ during its oscillatory phase.

Let $S(t)$ be the section of the surface $\Gamma$ for constant $t$ given by (3.1). From proposition 3.1 we deduce that the area $A(t, u(t))$ of $S(t)$ and the period $P(t, u(t))$ of the oscillation at the time $t$ are adiabatic invariants. Indeed we show that $A(t, u(t))$ is a first integral of equation (3.2) hence it is almost constant along the trajectory $\gamma(t)$. As $A(t, u(t))$ is given 
by

$$
A(t, u(t))=2 \int_{x_{1}(t, u)}^{x_{2}(t, u)} \sqrt{u-F(t, x)} d x
$$

and

$$
\frac{d A}{d t}(t, u(t))=\frac{\partial A}{\partial t}(t, u)+\frac{\partial A}{\partial u}(t, u) g(t, u)
$$

noticing that

$$
g(t, u)=-\frac{\frac{\partial A}{\partial t}(t, u)}{\frac{\partial A}{\partial u}(t, u)}
$$

we deduce $\frac{d A}{d t}(t, u(t))=0$.

Moreover since $P(t, u(t))=2 \frac{\partial A}{\partial u}(t, u(t))$ we have also

$$
\begin{aligned}
\frac{d P}{d t}(t, u(t)) & =\frac{\partial P}{\partial t}(t, u)+\frac{\partial P}{\partial u}(t, u) g(t, u) \\
& =2 \frac{\partial}{\partial u}\left(\frac{\partial A}{\partial t}(t, u)+\frac{\partial A}{\partial u}(t, u) g(t, u)\right) \\
& =0
\end{aligned}
$$

Hence the area and the period of oscillation are almost constant along trajectory $\gamma(t)$.

We can deduce the following result

Proposition 3.2. Let $\gamma(t)=(x(t), y(t))$ be the trajectory of (2.1) starting from $\left(t_{0}, x_{0}, y_{0}\right)$ in $O$ such that $A\left(t_{0}, u\left(t_{0}\right)\right)$ verifies

$$
\mathcal{A}\left(t_{*}\right)>A\left(t_{0}, u\left(t_{0}\right)\right)+O(\varepsilon)
$$

Then $\gamma(t)$ is a fast oscillating trajectory.

\section{Proof}

The area of the section, at constant $t$, of the region of oscillatory motion $O$ is given by

$$
\mathcal{A}(t)=2 \int_{x_{\sigma}(t)}^{x_{s}(t)} \sqrt{F\left(t, x_{s}(t)\right)-F(t, x)} d x
$$

$\mathcal{A}(t)$ is a $2 \pi$-periodic $C^{1}$ function and reachs its minimum at $t_{*} \in[0, \pi]$, besides the area $A(t, u(t))$ of the section at time $t$ of the cylinder $\Gamma$ approximating the graph of $\gamma(t)$ is constant, so $A(t, u(t))$ is equal to $A\left(t_{0}, u\left(t_{0}\right)\right)$ for all $t \in[0, \pi]$. Hence by $(3.7), \Gamma$ remains in $O$ for all $t \in[0, \pi]$

Periodicity and symmetry properties of system (2.1) lead to the same periodicity and symmetry properties of the surface $\Gamma$. Since the approximation of the graph of $\gamma(t)$ by the cylinder $\Gamma$ is of order $O(\varepsilon)$, 
trajectory $\gamma(t)$ remains in the oscillatory region $O$ for all time $t$.

In order to precise the position of the point $(x(t), y(t))$ of the fast oscillating trajectory $\gamma(t)$ on $\Gamma$ at a given $t$, we introduce and calculate, as in $[14,15]$, the number of rotations $N\left(t_{0}, t_{1}\right)$ completed by this trajectory around the center line $c(t)$ in the interval $\left[t_{0}, t_{1}\right]$.

Let

$$
\rho^{2}(t)=E(t, x(t), y(t))
$$

and for every $t$, consider the following change of variables along the trajectory $\gamma(t)$

$$
\left\{\begin{array}{l}
\rho \cos \theta=G(x) \\
\rho \sin \theta=y
\end{array}\right.
$$

where $G(x)$ is defined by

$$
G(x)=\left\{\begin{aligned}
-\sqrt{F(t, x)} & \text { for } \quad x<x_{c}(t) \\
\sqrt{F(t, x)} & \text { for } \quad x \geq x_{c}(t)
\end{aligned}\right.
$$

It easy to see that $G$ is smooth and $\frac{d G}{d x}(x)>0$ for all $x \neq x_{c}(t)$.

Definition 3.1. The rotation number, denoted by $\eta\left(t_{0}, t_{1}\right)$, of a solution with fast oscillating phase between times $t_{0}$ and $t_{1}$ is defined by

$$
\eta\left(t_{0}, t_{1}\right)=\frac{1}{2 \pi} \int_{t_{0}}^{t_{1}} \frac{d \theta}{d t}(s) d s
$$

Since trajectories turn clockwise around the center line $c(t)$, the number of rotations is given by $N\left(t_{0}, t_{1}\right)=-\eta\left(t_{0}, t_{1}\right)$.

As $u(t)$ is an approximation of $\rho^{2}(t)$, let us denote $r(t)=\sqrt{u(t)}$.

The following result provides an asymptotic estimate of the rotation number

Proposition 3.3. The rotation number of trajectory $\gamma(t)$ of system (2.1) with fast oscillating phase between times $t_{0}$ and $t_{1}$ is such that

$$
\eta\left(t_{0}, t_{1}\right)=-\frac{1}{\varepsilon} \int_{t_{0}}^{t_{1}} \frac{d t}{P(t, r(t))}+O(\varepsilon)
$$

where

$$
P(t, r(t))=2 \int_{0}^{\pi} \frac{r(t) \cos \varphi}{f(t, \xi(t, r(t), \varphi))} d \varphi
$$

and $\xi(t, r(t), \varphi)$ is the unique root of $G(x)=r(t) \cos \varphi$, 


\section{Proof}

Consider the derivative of $\theta(t)$ with respect to $t$; this derivative is well defined in the interval $\left[t_{0}, t_{1}\right]$ and given by

$$
\frac{d \theta}{d t}=\frac{d}{d t}\left(\arctan \frac{y(t)}{G(x(t))}\right)
$$

Straightforward calculation of the variations of $\theta(t)$ using the system (2.1) leads to

$$
\frac{d \theta}{d t}=\frac{-f(t, x(t))}{\varepsilon G(x(t))}-\frac{1}{2} \frac{y}{G(x(t))} \frac{\frac{\partial F}{\partial t}(t, x(t))}{y^{2}(t)+F(t, x(t))}
$$

Applying the change of variables (3.10) we obtain

$$
\varepsilon \frac{d \theta}{d t}=\frac{-f(t, x(t, \rho, \theta))}{\rho \cos \theta}-\frac{\varepsilon}{2} \frac{\sin \theta}{\cos \theta} \frac{\frac{\partial F}{\partial t}(t, x(t, \rho, \theta))}{\rho^{2}}
$$

where $x(t, \rho, \theta)$ is the unique root of $G(x(t))=\rho \cos \theta$.

Finally, we obtain the differential equation

$$
\varepsilon \frac{d \theta}{d t}=p(t, \rho, \theta)+\varepsilon q(t, \rho, \theta) \sin 2 \theta
$$

where $p$ and $q$ are given by

$$
\left\{\begin{array}{l}
p(t, \rho, \theta)=-\frac{f(t, x)}{\rho \cos \theta} \\
q(t, \rho, \theta)=-\frac{1}{4} \frac{\frac{\partial F}{\partial t}(t, x)}{\rho^{2} \cos ^{2} \theta}
\end{array}\right.
$$

To determine long time variations of the function $\theta(t)$ we shall use an averaging method. For that purpose, let $\left(t_{n}\right)_{n}$ be the sequence of successive instants such that $\theta$ varies by $2 \pi$. That is, if trajectory $\gamma(t)$ starts at the instant $t_{0}$ with the angle $\theta_{0}$, we have

$$
\theta\left(t_{n}\right)=\theta_{n}=\theta_{0}+2 n \pi
$$

Suppose given $\left(t_{n}, \theta_{n}\right)$, we shall compute the successive instant $t_{n+1}$. The change of variable and time

$$
\left\{\begin{aligned}
\Theta & =\frac{\theta-\theta_{n}}{\varepsilon} \\
T & =\frac{t-t_{n}}{\varepsilon^{2}}
\end{aligned}\right.
$$

applied to (3.15), leads to the differential equation

$$
\begin{aligned}
\frac{d \Theta}{d T}= & p\left(t_{n}+\varepsilon^{2} T, \rho\left(t_{n}+\varepsilon^{2} T\right), \theta_{n}+\varepsilon \Theta\right) \\
& +\varepsilon q\left(t_{n}+\varepsilon^{2} T, \rho\left(t_{n}+\varepsilon^{2} T\right), \theta_{n}+\varepsilon \Theta\right) \sin 2\left(\theta_{n}+\varepsilon \Theta\right)
\end{aligned}
$$


As long as $T$ is $O(1 / \varepsilon)$, solutions of this equation remain $\varepsilon^{2}$-close to those of the differential equation

$$
\frac{d \Theta}{d T}=p\left(t_{n}, \rho\left(t_{n}\right), \theta_{n}+\varepsilon \Theta\right)+\varepsilon q\left(t_{n}, \rho\left(t_{n}\right), \theta_{n}+\varepsilon \Theta\right) \sin 2\left(\theta_{n}+\varepsilon \Theta\right)
$$

Let $\Delta T_{n}$ the variation of time $T$ corresponding to the variation $\Delta \Theta=$ $\frac{2 \pi}{\varepsilon}$ of the angle $\Theta$ so that $\theta$ increases by $2 \pi$.

To compute $\Delta T_{n}$, we expand

$$
d T=\frac{d \Theta}{p\left(t_{n}, \rho\left(t_{n}\right), \theta_{n}+\varepsilon \Theta\right)+\varepsilon q\left(t_{n}, \rho\left(t_{n}\right), \theta_{n}+\varepsilon \Theta\right) \sin 2\left(\theta_{n}+\varepsilon \Theta\right)}
$$

up to order 2 in $\varepsilon$, and obtain

$$
\begin{aligned}
d T= & \frac{d \Theta}{p\left(t_{n}, \rho\left(t_{n}\right), \theta_{n}+\varepsilon \Theta\right)}\left[1-\varepsilon \frac{q\left(t_{n}, \rho\left(t_{n}\right), \theta_{n}+\varepsilon \Theta\right)}{p\left(t_{n}, \rho\left(t_{n}\right), \theta_{n}+\varepsilon \Theta\right)} \sin 2\left(\theta_{n}+\varepsilon \Theta\right)\right. \\
& \left.+\varepsilon^{2}\left(\frac{q\left(t_{n}, \rho\left(t_{n}\right), \theta_{n}+\varepsilon \Theta\right)}{p\left(t_{n}, \rho\left(t_{n}\right), \theta_{n}+\varepsilon \Theta\right)}\right)^{2} \sin ^{2} 2\left(\theta_{n}+\varepsilon \Theta\right)+O\left(\varepsilon^{3}\right)\right]
\end{aligned}
$$

Since the ratio of the functions $q$ and $p^{2}$ is an even function on $\theta$, the variation of time $\Delta T_{n}$ is given by

$$
\Delta T_{n}=\frac{2}{\varepsilon} \int_{0}^{\pi} \frac{d \Theta}{p\left(t_{n}, \rho\left(t_{n}\right), \Theta\right)}+2 \varepsilon \int_{0}^{\pi} \frac{q^{2}\left(t_{n}, \rho\left(t_{n}\right), \Theta\right)}{p^{3}\left(t_{n}, \rho\left(t_{n}\right), \Theta\right)} \sin ^{2} 2 \Theta d \Theta+O\left(\varepsilon^{2}\right)
$$

Hence $t_{n+1}=t_{n}+\varepsilon^{2} \Delta T_{n}$. Consider now the rate of variations of $\theta(t)$ with respect to $\left(t_{n}\right)$

$$
\begin{aligned}
& \frac{\theta\left(t_{n+1}\right)-\theta\left(t_{n}\right)}{t_{n+1}-t_{n}}=\frac{\Delta \Theta}{\varepsilon \Delta T_{n}} \\
& =\frac{\pi}{\varepsilon \int_{0}^{\pi} \frac{d \Theta}{p\left(t_{n}, \rho\left(t_{n}\right), \Theta\right)}+\varepsilon^{3} \int_{0}^{\pi} \frac{q^{2}\left(t_{n}, \rho\left(t_{n}\right), \Theta\right)}{p^{3}\left(t_{n}, \rho\left(t_{n}\right), \Theta\right)} \sin ^{2} 2 \Theta d \Theta}
\end{aligned}
$$

By expanding the above expression up to order 2 in $\varepsilon$ and using mean value theorem, we show that the solution $\theta(t)$ of equation (3.15) is $\varepsilon^{2}$-close to the solution of the differential equation

$$
\varepsilon \frac{d \theta}{d t}=\frac{\pi}{\int_{0}^{\pi} \frac{d \Theta}{p(t, r(t), \Theta)}}-\varepsilon^{2} 2 \pi \frac{\int_{0}^{\pi} \frac{q^{2}(t, r(t), \Theta)}{p^{3}(t, r(t), \Theta)} \sin ^{2} 2 \Theta d \Theta}{\int_{0}^{\pi} \frac{d \Theta}{p(t, r(t), \Theta)}}
$$

where we recall $r(t)=\sqrt{u(t)}$. Since equation (3.17) is $\theta$-independent, we find that the rotation number $\eta\left(t_{0}, t_{1}\right)$ of a solution with rapidly oscillating phase is of the form given by (3.13). 
The previous proposition shows that the rotation number $\eta\left(t_{0}, t_{1}\right)$ depends directly on the period $P(t, r(t))$. But as we have seen this period is an adiabatic invariant of trajectory $\gamma(t)$, hence period $P(t, r(t))$ depends at any time $t$ only on $r_{0}=r\left(t_{0}\right)$ (i.e. on the initial condition of trajectory $\gamma(t)$ ). So if this period of oscillation is monotone increasing with respect to $r_{0}$ then the number of rotations is monotone decreasing. We can state the following result on the monotonicity of the number of rotations. This result of monotonicity of the rotation number can be related to the condition of monotonicity of the period function of hamiltonian system exhibiting a center obtained by Chicone in [2].

Proposition 3.4. Let

$$
\Psi(t, x)=\frac{F(t, x)}{f(t, x)^{2}}
$$

and let $\gamma(t)$ be a trajectory of system (2.1) with rapidly oscillating phase between the times $t_{0}$ and $t_{1}$ starting from the point $\left(t_{0}, x_{0}, 0\right)$.

If $\frac{\partial^{2} \Psi}{\partial x^{2}}$ is positive in the region $O$ then the number of rotations $N\left(t_{0}, t_{1}\right)$ around $c(t)$ is a monotone decreasing function of the initial amplitude $\left|x_{0}-x_{c}\left(t_{0}\right)\right|$.

\section{Proof}

Since $\rho_{0}=G\left(x_{0}\right)$ and $\frac{d G}{d x}(x)>0, \rho_{0}$ increases with the amplitude $\left|x_{0}-x_{c}\left(t_{0}\right)\right|$. Hence, it suffices to show that the period $P\left(t_{0}, \rho_{0}\right)$ given by (3.14) is an increasing function with respect to $\rho_{0}$.

We drop the index of $\rho_{0}$ and compute the derivative $\frac{d P}{d \rho}$ of the period of a closed orbit of system (2.2) of energy $E(t, x, y)=\rho^{2}$.

By $\xi(t, \rho, \theta)=G^{-1}(\rho \cos \theta)$ we have

$$
\frac{\partial \xi}{\partial \rho}=-\frac{G(\xi(t, \rho, \theta))}{f(t, \xi(t, \rho, \theta))} \cos \theta
$$

We now differentiate $P\left(t_{0}, \rho\right)$ given by $(3.14)$ with respect to $\rho$

$$
\frac{d P}{d \rho}=2 \int_{0}^{\pi} \frac{\left(f\left(t_{0}, \xi\left(t_{0}, \rho, \theta\right)\right)\right)^{2}-\frac{\partial f}{\partial x}\left(t_{0}, \xi\left(t_{0}, \rho, \theta\right)\right) F\left(t_{0}, \xi\left(t_{0}, \rho, \theta\right)\right)}{\left(f\left(t_{0}, \xi\left(t_{0}, \rho, \theta\right)\right)\right)^{3}} \cos \theta d \theta
$$

Integrating by parts, where we put $u^{\prime}(\theta)=\cos (\theta)$ and

$$
v(\theta)=\frac{\left(f\left(t_{0}, \xi\left(t_{0}, \rho, \theta\right)\right)\right)^{2}-\frac{\partial f}{\partial x}\left(t_{0}, \xi\left(t_{0}, \rho, \theta\right)\right) F\left(t_{0}, \xi\left(t_{0}, \rho, \theta\right)\right)}{\left(f\left(t_{0}, \xi\left(t_{0}, \rho, \theta\right)\right)\right)^{3}}
$$

where the prime' ${ }^{\prime}$ is the derivative with respect to $x$, we obtain

$$
\frac{d P}{d \rho}=\rho \int_{0}^{\pi}\left(\frac{6\left(f^{\prime}\right)^{2} F-2 F f f^{\prime \prime}-6 f^{2} f^{\prime}}{f^{4}}\right)\left(t_{0}, \xi\left(t_{0}, \rho, \theta\right)\right) \frac{\sin ^{2} \theta}{G^{\prime}\left(\xi\left(t_{0}, \rho, \theta\right)\right)} d \theta
$$


Thus

$$
\frac{d P}{d \rho}=\rho \int_{0}^{\pi} \frac{\Psi^{\prime \prime}\left(\xi\left(t_{0}, \rho, \theta\right)\right)}{G^{\prime}\left(\xi\left(t_{0}, \rho, \theta\right)\right)} \sin ^{2} \theta d \theta
$$

Since $G^{\prime}$ is positive we conclude that $\frac{d P}{d \rho}$ is positive.

Consequently, proposition 3.3 shows that $\eta\left(t_{0}, t_{1}\right)$ is decreasing with respect to initial amplitude.

3.1. Trajectory in the vicinity of the center line. We can state, in a small tubular neighborhood of center line $c(t)$ of radius $\mathrm{O}(\sqrt{\varepsilon})$, analogue results as the previous ones concerning the adiabatic invariance of the action and the rotation number. For that purpose we perform the following change of variables

$$
\left\{\begin{array}{l}
X=\frac{x-x_{c}(t)}{\sqrt{\varepsilon}} \\
Y=\frac{y}{\sqrt{\varepsilon}}
\end{array}\right.
$$

System (2.1) becomes

$$
\left\{\begin{array}{l}
\varepsilon \frac{d X}{d t}=Y-\sqrt{\varepsilon} \frac{d x_{c}}{d t}(t) \\
\varepsilon \frac{d Y}{d t}=\frac{1}{\sqrt{\varepsilon}} f\left(t, x_{c}(t)+\sqrt{\varepsilon} X\right)
\end{array}\right.
$$

which can be rewritten as

$$
\left\{\begin{array}{l}
\varepsilon \frac{d X}{d t}=Y-\sqrt{\varepsilon} \frac{d x_{c}}{d t}(t) \\
\varepsilon \frac{d Y}{d t}=\frac{\partial f}{\partial x}\left(t, x_{c}(t)\right) X+\sqrt{\varepsilon} \frac{\partial^{2} f}{\partial x^{2}}\left(t, x_{c}(t)\right) X^{2}+O\left(\varepsilon^{2}\right)
\end{array}\right.
$$

As above the theorem of adiabatic invariance of the action in a Hamiltonian system depending slowly in time applied to (3.20) allows us to write the following

Proposition 3.5. Let $\sigma(t)=(X(t), Y(t))$ be the trajectory of system (3.20) of initial condition $\left(t_{0}, X_{0}, Y_{0}\right)$ and $\Sigma$ the surface of equation

$$
Y^{2}-\frac{\partial f}{\partial x}\left(t, x_{c}(t)\right) X^{2}=u_{0} \sqrt{\frac{\frac{\partial f}{\partial x}\left(t, x_{c}(t)\right)}{\frac{\partial f}{\partial x}\left(t_{0}, x_{c}\left(t_{0}\right)\right)}}
$$

where $u_{0}=Y_{0}^{2}+\frac{\partial f}{\partial x}\left(t_{0}, x_{c}\left(t_{0}\right)\right) X_{0}^{2}$.

Then $\sigma(t)$ verifies

$$
Y(t)^{2}-\frac{\partial f}{\partial x}\left(t, x_{c}(t)\right) X(t)^{2}=u_{0} \sqrt{\frac{\frac{\partial f}{\partial x}\left(t, x_{c}(t)\right)}{\frac{\partial f}{\partial x}\left(t_{0}, x_{c}\left(t_{0}\right)\right)}}+O(\varepsilon)
$$

Moreover the area of the section, at constant $t$, of $\Sigma$ is a constant function with respect to time $t$. 
We can also obtain, in the tubular neighborhood of the center line $c(t)$, an analogous result to proposition 3.3, the reader can found in [13] a detailed proof of the following

Proposition 3.6. The rotation number of a trajectory $\sigma(t)$ of system (3.20) starting at $t_{0}$ is such that

$$
\eta\left(t_{0}, t\right)=-\frac{1}{\varepsilon} J\left(t_{0}, t\right)+O(\varepsilon)
$$

where

$$
J\left(t_{0}, t\right)=\frac{1}{2 \pi} \int_{t_{0}}^{t} \sqrt{-\frac{\partial f}{\partial x}\left(s, x_{c}(s)\right)} d s
$$

This last result shows that the motion in a small tubular neighborhood of center line $c(t)$ is quasi-isochronous since the rotation number is approximated by $J\left(t_{0}, t\right)$ which does not depend on the initial conditions of the trajectory in the vicinity of the center line.

\section{Proof of Theorems}

We have seen in section 2 that, as long as the variation of $t$ is small of order $\varepsilon$, the graph of the trajectory of (2.1) with initial condition $\left(x_{0}, 0\right)$ in the region $\Omega$ is approximated by the unbounded curve defined by equation (2.6). System (2.1) shows that the components $x(t)$ and $y(t)$ are positive increasing functions. So we can state the following result

Proposition 4.1. Let $\gamma(t)=(x(t), y(t))$ be a trajectory of (2.1) starting from a point $\left(t_{0}, x_{0}, 0\right)$ of the $x$-axis in $\Omega$, hence components $x(t)$ and $y(t)$ are monotone increasing functions in $\left[t_{0},+\infty\right)$ and for sufficiently large $t, \gamma(t)$ remains in the quarter-plan $\mathbb{R}^{+} \times \mathbb{R}^{+}$.

Before giving the proof of theorem 2.1, let us construct the nonlinear resonance values $\varepsilon_{n}$. According to proposition 3.6, the motion in the vicinity of the center line $c(t)$ is quasi-isochronous. So, suppose a trajectory realizes a whole number $n$ of half-rotations in the interval of time $[0, \pi]$ hence its rotation number $\eta(0, \pi)$ is equal to $-n / 2$ (recall that the solution turns clockwise around $c(t))$. The rotation number given by equation $(3.22)$ is

$$
-\frac{n}{2}=\eta(0, \pi)=-\frac{1}{\varepsilon} J(0, \pi)+\varepsilon C
$$

Now, compute $\varepsilon$ with respect to $n$ we have

$$
\varepsilon=\frac{-n+n \sqrt{1+\frac{16 J(0, \pi) C}{n^{2}}}}{4 C}=\frac{2 J(0, \pi)}{n}+O\left(\frac{1}{n^{2}}\right)
$$


and finaly

$$
\varepsilon_{n}=\frac{2 J(0, \pi)}{n}
$$

Now we are able to give the proofs of our main results.

Proof of theorem 2.1

Denote by $\Pi$, the Poincaré map of half-period associated to system (3.20) defined by

$$
\begin{array}{ll}
\Pi: \mathbb{R}^{2} & \rightarrow \mathbb{R}^{2} \\
\left(X_{0}, Y_{0}\right) & \mapsto(X(\pi), Y(\pi))
\end{array}
$$

where $(X(t), Y(t))$ is the trajectory of system (3.20) starting at $t=0$ from the point $\left(X_{0}, Y_{0}\right)$.

Consider $\Pi$ the image of the $X$-axis by map $\Pi$ defined by $\Pi_{X}=$ $\left\{\Pi\left(X_{0}, 0\right) \in \mathbb{R}^{2} / X_{0} \in \mathbb{R}\right\}$ and let $\varepsilon$ a small parameter such that hypothesis of theorem 2.1 holds. According to proposition 3.6, since $\varepsilon$ is far away from resonant values $\varepsilon_{n}$, the image $\Pi_{X}$ is approximated by a straight line passing by the origin and different from the $X$-axis. Hence $\Pi_{X}$ crosses the $X$-axis at some point $X_{\pi}$, by continuity of $\Pi_{X}$ there is an input $\bar{X} \in \mathbb{R}$ such that $\Pi(\bar{X}, 0)=\left(X_{\pi}, 0\right)$. Let $\Gamma(t)=(X(t), Y(t))$ the trajectory of system (3.20) starting from $(\bar{X}, 0)$, it obviously verifies $Y(\pi)=0$. It is clear that $\bar{X}$ is $O(\sqrt{\varepsilon})$. In the coordinates $(x, y)$, the corresponding solution $(\xi(t), \zeta(t))$ starting at $t=0$ from the point $(\bar{x}, 0)$ with $\bar{x}=x_{c}(0)+\sqrt{\varepsilon} \bar{X}$ is such that $\zeta(\pi)=0$ hence by symmetry $\xi(t)$ is an even $2 \pi$-periodic solution of equation (1.2).

Let us show now that this solution is unique. Consider the variational differential system associated to the periodic trajectory $(\xi(t), \zeta(t))$

$$
\left\{\begin{array}{l}
\varepsilon \frac{d U}{d t}=V \\
\varepsilon \frac{d V}{d t}=-\frac{\partial f}{\partial x}(t, \xi(t)) U
\end{array}\right.
$$

Since $\xi(t)$ is close to $x_{c}(t), \frac{\partial f}{\partial x}(t, \xi(t))$ is strictly negative. System (4.2) is of the same type as system (3.20) we can apply the same argument as above to see that the image by the Poincaré map $\Pi$ of the $X$-axis is a straight line passing throw the origin and different from the $X$-axis. So system (4.2) can not admit a $2 \pi$-periodic non trivial solution.

Hence, system (4.2) has no non trivial $2 \pi$-periodic solution in the tubular neighborhood of $c(t)$ of radius $O(\sqrt{\varepsilon})$. We conclude by Poincaré perturbation theorem [5, p. 415].

\section{Proof of theorem 2.3.}

To prove existence of the finite family $\left(\varphi_{n, i}\right)$ of $x$-symmetric $2 \pi$-periodic 
trajectories of system $(2.1)$, we consider the interval $I_{a}=\left[a_{-}, a_{+}\right]$containing the interval $I_{0}=\left[x_{\sigma}(0), x_{s}(0)\right]$ such that $a_{-}$et $a_{+}$are respectively not $\varepsilon$-close to $x_{\sigma}(0)$ and $x_{s}(0)$ so that $\left(0, a_{-}, 0\right)$ and $\left(0, a_{+}, 0\right)$ are in the region $\Omega$ of unbounded motion. Consider the image of the segment $I_{a} \times\{0\}$ by the Poincaré map of half-period $P$ associated to system (2.1) defined by

$$
\begin{array}{rlll}
P: & \mathbb{R}^{2} & \rightarrow \mathbb{R}^{2} \\
& \left(x_{0}, y_{0}\right) & \mapsto(x(\pi), y(\pi))
\end{array}
$$

where $(x(t), y(t))$ is the trajectory of system (2.1) starting at $t_{0}=0$ from the point $\left(x_{0}, y_{0}\right)$.

According to proposition $4.1, P\left(a_{-}, 0\right)$ and $P\left(a_{+}, 0\right)$ remain in $\mathbb{R}^{+} \times \mathbb{R}^{+}$ hence trajectories of $(2.1)$ starting from the points $\left(a_{-}, 0\right)$ and $\left(a_{+}, 0\right)$ at time $t_{0}=0$ realize in clockwise direction respectively less than one half-rotation and less than one quarter-rotation around the center line $c(t)$.

On the other hand, according to proposition 3.6, for all point $x_{0} \in I_{0}$ in the vicinity of $x_{c}(0)$, the number of rotations $N(0, \pi)$ of the trajectory of system (2.1) starting from $\left(x_{0}, 0\right)$ at $t_{0}=0$ is of order $O(1 / \varepsilon)$. Since system (2.1) has continuity property of trajectories with respect to initial conditions, $N(0, \pi)$ is a continuous function with respect to the amplitude $\left|x_{0}-x_{c}(0)\right|$, moreover, by proposition 3.4, this function is monotone decreasing. Its maximum $N_{\varepsilon}$, given by (2.9), is reached in the vicinity of $x_{c}(0)$. Then by continuity of application $P$, there exist two positive numbers $\alpha_{+}$and $\alpha_{-}$such that for all integer $n$, with $0 \leq n \leq N_{\varepsilon}$, there exists a unique point $\xi_{n, 0}^{0} \in\left[x_{c}(0), x_{s}(0)+\varepsilon \alpha_{+}\right]$ (respectively $\xi_{n, 1}^{0} \in\left[x_{\sigma}(0)-\varepsilon \alpha_{-}, x_{c}(0)\right]$ ) such that the number of rotations of the trajectory $\varphi_{n, 0}(t)=\left(\xi_{n, 0}(t), \zeta_{n, 0}(t)\right)$ (respectively $\varphi_{n, 1}(t)=$ $\left.\left(\xi_{n, 1}(t), \zeta_{n, 1}(t)\right)\right)$ starting from $\left(\xi_{n, 0}^{0}, 0\right)$ (respectively $\left.\left(\xi_{n, 1}^{0}, 0\right)\right)$ is equal to $\frac{n}{2}$ (respectively $\frac{n+1}{2}$ ).

In the half-period, this trajectory realizes a whole number of halfrotations around the center line $c(t)$ so its component $\zeta_{n, i}(t)$ vanishes at $t=\pi$. Since this trajectory is $\mathrm{x}$-symmetric, it is $2 \pi$-periodic.

\section{Proof of theorem 2.2.}

Recall that $u_{*}$ is the level of energy integral of the homoclinic separatrix so that its area reaches its minimum at $t_{*}$. Consider as in the previous poof, the image by Poincaré map (4.3) of the segment $\left[x_{1}\left(0, u_{*}\right), x_{2}\left(0, u_{*}\right)\right] \times\{0\}$ include in $O$.

According to proposition 3.2, trajectories of (2.1) starting from points of $\left[x_{1}\left(0, u_{*}\right), x_{2}\left(0, u_{*}\right)\right] \times\{0\}$ are fast oscillating trajectories. The number of rotation $N(0, \pi)$, at the bounds of this segment, is equal to $n_{\varepsilon}$, 
while in the vicinity of center $c(0)$ it is equal to $N_{\varepsilon}$, given by (2.9). Since, by proposition 3.4, the number of rotation is a decreasing function we conclude, as in the previous proof, the existence of rapidly oscillating $2 \pi$-periodic even solutions that have $n$ oscillations by period for each $n \in\left[n_{\varepsilon}, N_{\varepsilon}\right]$.

\section{Application and Numerical Illustration}

In this section, we shall apply our results to the different examples given in the introduction. We can apply at each equation theorems 2.1 , 2.2 and 2.3. To do so, we can verify easily hypotheses (2.1) and (2.1) for these equations:

For pendulum equation (1.3) and Duffing equation (1.4), the phase portrait has an other symmetry plan at $t=\frac{\pi}{2}$. Hypothesis (2.1) is verified and there exists an oscillatory region defined for all $t \in \mathbb{R}$ if we choose $0<\beta<1$ in equation (1.3). The same occurs for equation (1.4) if we choose $a>0, b<0$ and $0<\beta<\frac{2}{3} a \sqrt{-\frac{a}{3 b}}$.

For these equations, the area $\mathcal{A}(t)$ is an increasing function in the interval $\left[0, \frac{\pi}{2}\right]$, hence we have

$$
\forall t \in[0, \pi], \quad \mathcal{A}\left(\frac{\pi}{2}\right) \geq \mathcal{A}(t) \geq \mathcal{A}(0)>0
$$

For the "shallow water sloshing" problem, if we choose $\lambda>0$ in equation (1.5), hypothesis (2.1) is verified and there exists also an oscillatory region defined for all $t \in \mathbb{R}$. For this equation, the area $\mathcal{A}(t)$ is a decreasing function in $[0, \pi]$ and verifies

$$
\forall t \in[0, \pi], \quad \mathcal{A}(0) \geq \mathcal{A}(t) \geq \mathcal{A}(\pi)>0
$$

Let us verify that hypothesis (2.2) is also satisfied by our examples which has the consequence that the number of rotation is a decreasing function of the initial amplitude $\left|x(0)-x_{c}\right|$. This explains the fact that more and more $2 \pi$-periodic trajectories appear as $\omega$ increases. These trajectories are classified by their numbers of rotation by period around the center line $c(t)$. We compute the seconde derivative $\frac{\partial^{2} \Psi}{\partial x^{2}}$ where $\Psi(t, x)=\frac{F(t, x)}{f(t, x)^{2}}$ and verify that it is positive.

Since the period of oscillation is an adiabatic invariant, we can compute this derivative for any fixed $t$. If we choose $t=\pi / 2$ then $x_{c}(\pi / 2)=0$ for equations (1.3) and (1.4) which leads to a simpler expression of $F(t, x)$. 
Forced pendulum : For the forced pendulum equation we have $\Psi(\pi / 2, x)=\frac{2(1-\cos (x))}{\sin ^{2}(x)}$ hence straightforward computation conduces to

$$
\frac{\partial^{2} \Psi}{\partial x^{2}}(\pi / 2, x)=\frac{2(1-\cos (x))^{2}(2-\cos (x))}{\sin ^{4}(x)}
$$

which is positive for all $x$.

Duffing equation : For this equation we have $\Psi(\pi / 2, x)=$ $\frac{2 a x^{2}+b x^{4}}{2\left(a x+b x^{3}\right)^{2}}$ and obtain

$$
\frac{\partial^{2} \Psi}{\partial x^{2}}(\pi / 2, x)=3 \frac{b x^{4}\left(-a^{2}+4 a b x^{2}+b^{2} x^{4}\right)}{\left(a x+b x^{3}\right)^{4}}
$$

The sign of $\frac{\partial^{2} \Psi}{\partial x^{2}}(\pi / 2, x)$ depends on the sign of parameters $a$ and $b$. For example, if we choose $a>0, b<0$ and $\beta>0$ such that $\beta<\frac{2}{3} a \sqrt{-\frac{a}{3 b}}$ then there is an oscillatory region defined for all $t \in \mathbb{R}$. Hence for $a>0$ and $b<0$, the derivative $\frac{\partial^{2} \Psi}{\partial x^{2}}(\pi / 2, x)$ is positive in the interval $\left[-\sqrt{2+\sqrt{5}} \sqrt{-\frac{a}{b}}, \sqrt{2+\sqrt{5}} \sqrt{-\frac{a}{b}}\right]$ which includes the oscillatory interval $\left[-\sqrt{-\frac{a}{b}}, \sqrt{-\frac{a}{b}}\right]$.

Shallow water sloshing problem : Let some fixed $\bar{t}$ and denote $\bar{x}_{c}=x_{c}(\bar{t})=-\sqrt{1+\lambda+\cos (\bar{t})}$. The change of variable $X=x-\bar{x}_{c}$ in equation (1.5) leads to the equivalent equation

$$
\varepsilon^{2} \frac{d^{2} X}{d t^{2}}=2 \bar{x}_{c} X+X^{2}
$$

For this last equation, we have $\Psi(\bar{t}, X)=-\frac{2}{3} \frac{3 \bar{x}_{c} X^{2}+X^{3}}{\left(2 \bar{x}_{c} X+X^{2}\right)^{2}}$ and obtain

$$
\frac{\partial^{2} \Psi}{\partial X^{2}}(\bar{t}, X)=-\frac{4}{3} \frac{X^{4}\left(5 \bar{x}_{c}+X\right)}{\left(2 \bar{x}_{c} X+X^{2}\right)^{4}}
$$

The trajectory remains in the oscillatory region if $X \in\left[X_{\sigma}, X_{s}\right]=$ $\left[\bar{x}_{c},-\bar{x}_{c}\right]$ and in this interval $\frac{\partial^{2} \Psi}{\partial X^{2}}(\bar{t}, X)$ is positive.

We conclude this study by a numerical analysis of the structure of the even $2 \pi$-periodic solutions of equation (1.5).

We have plot, in figure 3 , the response curves diagram of equation (1.5) with $\lambda=5$. We retrieve the unique solution predicted by theorem 2.1 near the center. When $\omega$ crosses the nonlinear resonant values $\omega_{n}$ a 
bifurcation appears and gives rise to two new branches of periodic solutions which have $n$ oscillations by period. These branches correspond to initial conditions of rapid oscillating trajectories predicted by theorem 2.2 when these initial conditions are far from $x_{s}$ and $x_{\sigma}$ and to initial conditions of phase oscillating trajectories predicted by theorem 2.3 with 0 or 1 spike near $t=\pi$

We can see when $\omega$ increases ( $\varepsilon$ decreases), like observed in $[6,11]$ for equation (1.5) (and also in [10, 15] for equation (1.3), in [17] for equation (1.4) see figure 3), that more and more initial conditions appear. In fact at each time, when $\omega$ crosses the nonlinear resonance values $\omega_{n}=1 / \varepsilon_{n}$, a new branch of periodic solutions borns. This new branch wins one rotation around the center line $c(t)$. Moreover near $x_{c}(0)$ but when $\omega$ is far from $\omega_{n}$, according to theorem 2.1, there is an unique initial condition of periodic solution.

In figure 4 we have plotted some of these solutions obtained for $\omega_{*}=4$ $\left(\varepsilon_{*}=0.25\right)$ and $\lambda=5$, figure 4 -(a) represents the solutions of the family $\xi_{n, 0}$ for $n=1$ to $n=8$ and figure 4 -(b) represents the solutions of the family $\xi_{n, 1}$ for $n=0$ to $n=7$.

We have also two other solutions that we have not plotted here, the solution $\xi_{0,0}$ whose the graph is closed to the graph of $x_{s}$ (dashed line) and the solution $\xi$ predicted by theorem 2.1 whose the graph is closed to the graph of the center $x_{c}$ (dot-dashed line)

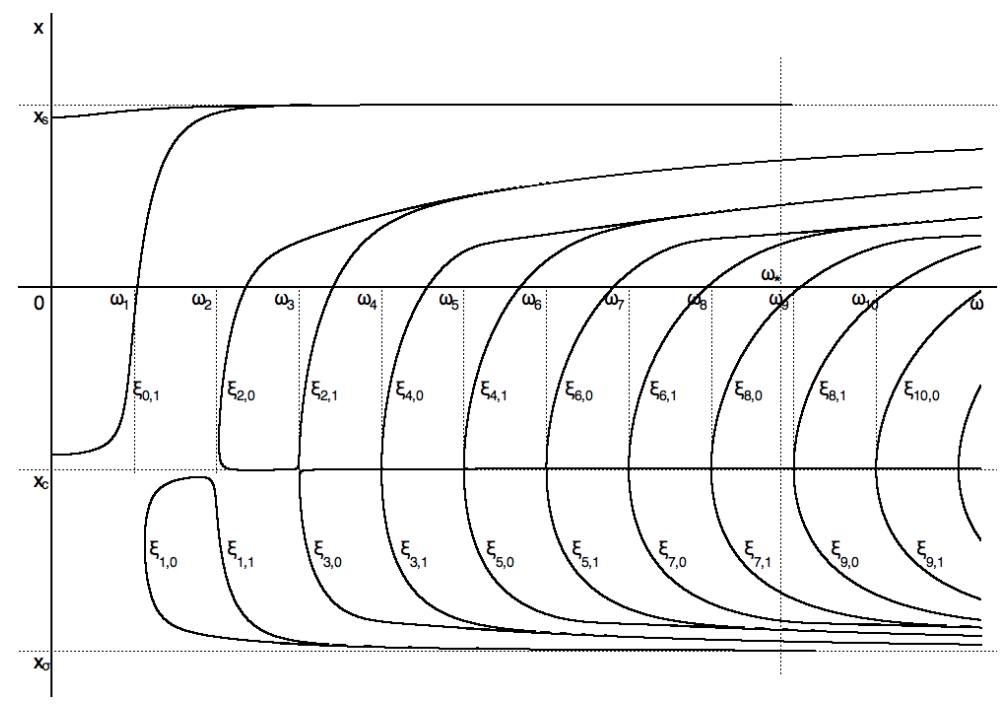

FiguRE 3. Response curves of equation 1.5 with respect to the parameter $\omega=1 / \varepsilon$. 


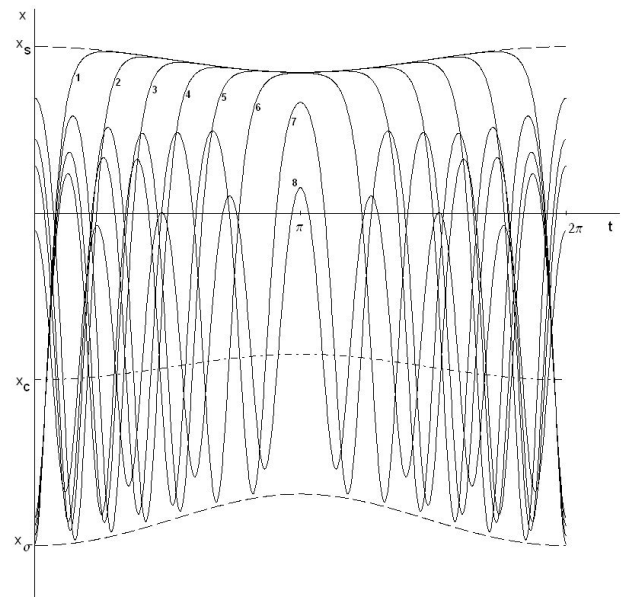

(a)

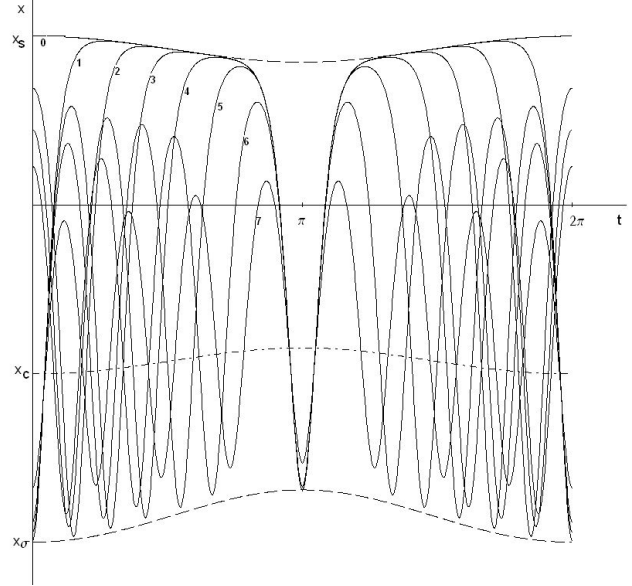

(b)

FiguRE 4. $2 \pi$-periodic solutions obtained for $\omega=4$ and $\lambda=5$, the number corresponds to $n$ in the family $\xi_{n, i}$ (a) $i=0$ spike near $t=\pi$ and (b) $i=1$ spike near $t=\pi$.

\section{REFERENCES}

[1] Ai, S., Hastings, S. P.(2002). A shooting approach to layers and chaos in a forced Duffing equation. Journal of Differential Equations. 185, 389-436.

[2] Chicone, C. (1987). The monotonicity of the period function of planar Hamiltonian vector fields. Journal of Differential Equations. 69 , 310-321.

[3] Fenichel, N. (1979). Geometric Singular Perturbation Theory. Journal of Differential Equations. 31, 53-98.

[4] Gedeon, T., Kokubu, H., Mischaikow, K., and Oka, H. (2002). Chaotic solutions in slowly varying perturbations of Hamiltonian systems with application to shallow water sloshing. Journal of Dynamics and Differential Equations. $14,63-84$.

[5] Hartmann, P. (1964). Ordinary Differential Equation. Willey, New York.

[6] Hastings, S.P., McLeod, J.B. (1991). On the periodic solutions of a forced second-order equation. J. NonLinear Sci. 1, 225-245.

[7] Jones, C.K.R.T. (1994). Geometric Singular Perturbation Theory. In Dynamical Systems, Montecatini Terme, Lecture Notes in Mathematics, 1609.

[8] Mawhin, J. (1998). Seventy-five years of global analysis around the forced pendulum equation. In Proceedings of Equadiff 9, Conference on Differential Equations and Their Applications, Brno, August 25-29, 1997, [Part 1] Survey papers (Editors: R. P. Agarwal; F. Neuman and J. Vosmanskỳ) Masaryk University, Brno, 115-145.

[9] Mawhin, J. (2010). Periodic solutions of the forced pendulum: classical vs relativistic. Matematiche (Catania) 65 no. 2, 97-107.

[10] Meissner, L.P. (1969). High order resonance for Duffing's differential equation. SIAM J. Appl. Math. 17, 240-250. 
[11] Ockendon, H., Ockendon, J.R., and Johnson, A.D. (1986). Resonant sloshing in shallow water. J. Fluid Mech. 167, 465-479.

[12] Oka, H. (1993). Singular perturbations of autonomous ordinary differential equations and heteroclinic bifurcations. In Dynamical Systems, Santiago 1990, Pitman Research Notes Math., 285, Longman.

[13] Sari, N. (1994). Oscillations non linéaires avec symétries périodiquement et lentement forcées. Bulletin de la Classe des Sciences, Académie Royale de Belgique. 5, 317-343.

[14] Sari, N. (2001). Periodic solutions of singularly nonlinear differential equations with symmetries. Dyn. Cont. Disc. Impuls. Sys. Ser. A. 8, 305-333.

[15] Sari, N., Schmitt, B.V. (1991). Oscillations lentement forcées du pendule simple. J. Appl. Math. Phys. 14, 136-141.

[16] Sari, T. (1995). Stroboscopy and Averaging. In Colloque trajectorien à la mémoire de J.L. Callot et G. Reeb. Strasbourg-Obernai 1995, Publication IRMA, Univ. Strasbourg. 125-158.

[17] Schmitt, B.V. (1982). Sur la structure de l'équation de Duffing sans dissipation. SIAM Journal of Applied Mathematics. 42, 868-894.

[18] Schmitt, B.V., Mazzanti, S. (1981). Solutions périodiques symétriques de l'équation de Duffing sans dissipation. Journal of Differential Equations. 42, 199-214.

[19] Stoker, J.J. (1950). Nonlinear vibrations in mechanical and electrical systems, Intersciences, New-York.

[20] Szmolyan, P. (1991). Transversal Heteroclinic and Homoclinic orbits in Singularly Perturbed Problems. Journal of Differential Equations. 92, 252-281. 